See discussions, stats, and author profiles for this publication at: https://www.researchgate.net/publication/279961021

Tracking futsal players with a wide-angle lens camera: accuracy analysis of the radial distortion correction based on an improved Hough transform algorithm

Article · March 2017

DOI: $10.1080 / 21681163.2015 .1072055$

CITATIONS

READS

8

847

9 authors, including:

(2) Luiz Henrique Palucci Vieira

São Paulo State University

35 PUBLICATIONS 69 CITATIONS

SEE PROFILE

2. Ricardo Augusto Barbieri

1. São Paulo State University

34 PUBLICATIONS 83 CITATIONS

SEE PROFILE

Some of the authors of this publication are also working on these related projects:

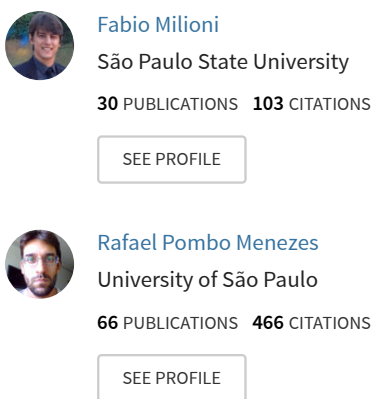

Proyecto Aorta View project

SAR/PoISAR View project 


\section{Tracking futsal players with a wide-angle lens camera: accuracy analysis of the radial distortion correction based on an improved Hough transform algorithm}

Luiz H. P. Vieira, Emilio A. Pagnoca, Fabio Milioni, Ricardo A. Barbieri, Rafael P. Menezes, Luis Alvarez, Luis G. Déniz, Daniel Santana-Cedrés \& Paulo R. P. Santiago

To cite this article: Luiz H. P. Vieira, Emilio A. Pagnoca, Fabio Milioni, Ricardo A. Barbieri, Rafael P. Menezes, Luis Alvarez, Luis G. Déniz, Daniel Santana-Cedrés \& Paulo R. P. Santiago (2017) Tracking futsal players with a wide-angle lens camera: accuracy analysis of the radial distortion correction based on an improved Hough transform algorithm, Computer Methods in Biomechanics and Biomedical Engineering: Imaging \& Visualization, 5:3, 221-231, DOI: 10.1080/21681163.2015.1072055

To link to this article: http://dx.doi.org/10.1080/21681163.2015.1072055

Published online: 20 Aug 2015.

Submit your article to this journal $\llbracket$

Џ Article views: 99

View related articles $₫$

View Crossmark data $\nearrow$ 


\title{
Tracking futsal players with a wide-angle lens camera: accuracy analysis of the radial distortion correction based on an improved Hough transform algorithm
}

\author{
Luiz H. P. Vieiraa, Emilio A. Pagnocab, Fabio Milionic, Ricardo A. Barbieric, Rafael P. Menezes ${ }^{a}$, Luis Alvarez ${ }^{\mathrm{d}}$, Luis G. Déniz ${ }^{\mathrm{d}}$, \\ Daniel Santana-Cedrés ${ }^{\mathrm{d}}$ and Paulo R. P. Santiago ${ }^{\mathrm{a}}$ \\ aLaboratório de Biomecânica e Controle Motor, School of Physical Education and Sport of Ribeirão Preto, University of São Paulo, Ribeirão Preto, Brazil; \\ ${ }^{b}$ Faculty of Pharmaceutical Sciences of Ribeirão Preto, University of São Paulo, Ribeirão Preto, Brazil; ' Institute of Biosciences, São Paulo State University \\ "Júlio de Mesquita Filho", Rio Claro, Brazil; 'Departamento de Ingeniería Electrónica y Automática, CTIM (Image Technology Center), Universidad de Las \\ Palmas de Gran Canaria, Las Palmas de Gran Canaria (Canary Islands), Spain
}

\begin{abstract}
The aim of this study was to assess the applicability and reliability of a single wide-angle lens GoPro camera for tracking and kinematics analysis of futsal players. An official game of a Brazilian professional team in the quarter-final round of the 2013 São Paulo futsal league was recorded by four digital video cameras ( $30 \mathrm{~Hz}$; $720 \times 480)$ placed at the highest points of the court $(40 \times 20 \mathrm{~m}$; FIFA standard). We compared tracking performed with three cameras equipped with conventional lenses to a GoPro ${ }^{\circledR}$ camera with a wide-angle lens with $170^{\circ}$ field of view. Manual and automatic tracking of players trajectories $(n=5 ; 23.20 \pm 2.39$ years $)$ during competition and in a controlled environment were performed. We found that the root-mean-square error calculated to determine the position on the court based on images captured with a GoPro ${ }^{\oplus}$ camera was $0.32 \mathrm{~m}$ and for the velocity was $0.71 \mathrm{~m} \mathrm{~s}^{-1}$. A 5-min excerpt of the game showed an average difference of $2.11 \%$ between the total distance covered obtained by tracking with the GoPro ${ }^{\circledast}$ with radial distortion correction (438.97 $\pm 164.65 \mathrm{~m}$ ), and cameras with conventional lenses (449.54 $\pm 170.92 \mathrm{~m}$ ). Temporal correlation analysis confirmed the great similarity between the reconstructed trajectories obtained by these cameras ( $r=0.99$ for movements on both $x$ and $y$ axes). We showed that a single GoPro ${ }^{\oplus}$ Hero3+ can provide reliable kinematic analysis of computational tracking based on videogrammetry for futsal, using the Hough transform and division model technique for radial distortion correction.
\end{abstract}

\section{ARTICLE HISTORY}

Received 30 November 2014

Accepted 9 July 2015

\section{KEYWORDS}

Radial distortion; Hough transform; GoPro; automatic tracking; futsal; biomechanics

\section{Introduction}

Generally speaking, studies that analyse team sports performance use video cameras to investigate kinematic variables of motion by means of image processing. By computational tracking, it is possible to identify the total distances covered, range of velocities and kinematical analysis of player movement trajectories or collective coordination during competition (Barros et al. 2007, 2011; Barbero-Alvarez et al. 2008; Bueno et al. 2014; Vilar et al. 2014).

In indoor sports, such as swimming, basketball and futsal, there are physical limitations (walls, roofs, bleachers) on the use of conventional cameras that are able to frame the whole venue for further computational tracking, as it is necessary to move the cameras backwards and it is not always possible to get the appropriate distance in such venues (Pers et al. 2008). Therefore, it is often necessary to use more than one camera or device in order to obtain the desired data, which increases the amount of time and resources required, and in some cases, this may be impossible. A very common solution is the use of wide-angle spherical lenses, as they include more of the scene due to their wider field of view, which can be up to $180^{\circ}$, and they are capable of capturing the whole scene, even when they are close to the object (Pers et al. 2008).

Progress in the field of computer vision techniques with the use of cameras equipped with wide-angle lenses for motion capture by means of tracking in the context of sports is the result of the work of Pers and colleagues (Pers et al. 2002, 2008; Perše et al. 2009; Martínez-Gallego et al. 2013). There are reports of studies that used wide-angle lens cameras to obtain motion variables during tennis (Martínez-Gallego et al. 2013), basketball (Perše et al. 2009), handball (Pers et al. 2002) and squash games (Vučkovič et al. 2010). Radial distortion correction of image sequences was achieved using a nonparametric exponential function instead of the traditional polynomial approximation models (Pers \& Kovacic 2002; Pers et al. 2008).

Few correction models, except for the nonparametric barrel distortion correction model of Pers and Kovacic (2002), have been used in a sports context for computational tracking (see also Pers et al. 2008). A recent review reported that the best radial distortion model for cameras with high degrees of distortion is the division model (Alemán-Flores et al. 2013). It is important to note that image distortion of wide-angle cameras strongly 
affects post-processing operations, it is very important to know this when we deal with sports events monitoring such as computational tracking, which requires the use of very accurate models. However, the division model has not been widely applied to image processing for tracking.

For futsal and many other team sports, a single camera with a wide-angle lens (CWAL) has not yet been widely used. Previous research on court team sports has used at least two cameras (Pers et al. 2002; Perše et al. 2009). A great number of studies involving computational tracking specifically for futsal have used at least two (Barbero-Alvarez et al. 2008; Bueno et al. 2014), and sometimes four (Moura et al. 2012; Morais et al. 2014) conventional lens cameras to obtain variables of interest, due to the difficulty of capturing the whole court scene with cameras with this type of lens, which tends to result in high costs of image storage and processing time (Barros et al. 2007, 2011; Perše et al. 2009). For example, despite a playing time of $40 \mathrm{~min}$ (two 20-min halves) as established by futsal game rules (FIFA 2014), an official futsal game extends over $75-85 \%$ than the scheduled (Barbero-Alvarez et al. 2008). When cameras with a standard acquisition frequency of $30 \mathrm{~Hz}$ are used, a futsal game yields 144,000 frames per camera. Therefore, reducing the number of cameras to a minimum is essential to perform tracking analysis more quickly (see for instance, Carling et al. 2008).

In addition, the use of a single camera, as opposed to multiple cameras, has been proposed for team sports tracking, since it is more cost-effective (Pers et al. 2002; Toki \& Sakurai 2005). Studies that have used a single camera for tracking soccer have obtained only partial results for errors associated with the use of a single camera (as in Toki \& Sakurai 2005), and the radial distortion correction model has only been partially reported when wide-angle lenses are used (as in Clemente et al. 2014). In this way, errors associated with the use of a single camera for computational tracking analysis are as yet unknown (Carling et al. 2008).

Therefore, the aim of this study was to assess the applicability and reliability of a single wide-angle lens camera, with barrel distortion images corrected by an improved Hough transform algorithm that includes the division model (Alemán-Flores et al. 2014), by comparing it with the use of three conventional lens cameras in the kinematic analysis of computational tracking in futsal.

\section{Methods}

\section{Participants and data collection}

An official futsal game of a Brazilian professional team in the quarter-final round of the 2013 São Paulo futsal league was recorded in full by four digital video cameras placed at the highest points of the court. They were set to an acquisition frequency of $30 \mathrm{~Hz}(720 \times 480$ and 24-bit colour resolution); three were cameras with conventional lenses (CCL) (SONY ${ }^{\mathrm{TM}}$ DCD-SR21) and one was a CWAL (GoPro ${ }^{\circledR}$ Hero3+ Black Edition, Woodman Labs Inc., USA) that captured the whole court (Figure 1).

An excerpt of the game was selected, in which five players were monitored ( $23.20 \pm 2.39$ years; $73.78 \pm 6.05 \mathrm{~kg} ; 173.60 \pm 6.05 \mathrm{~cm}$ ). Procedures complied with the terms of the local Research Ethics Committee (protocol no. 5069 of 18/07/2012).
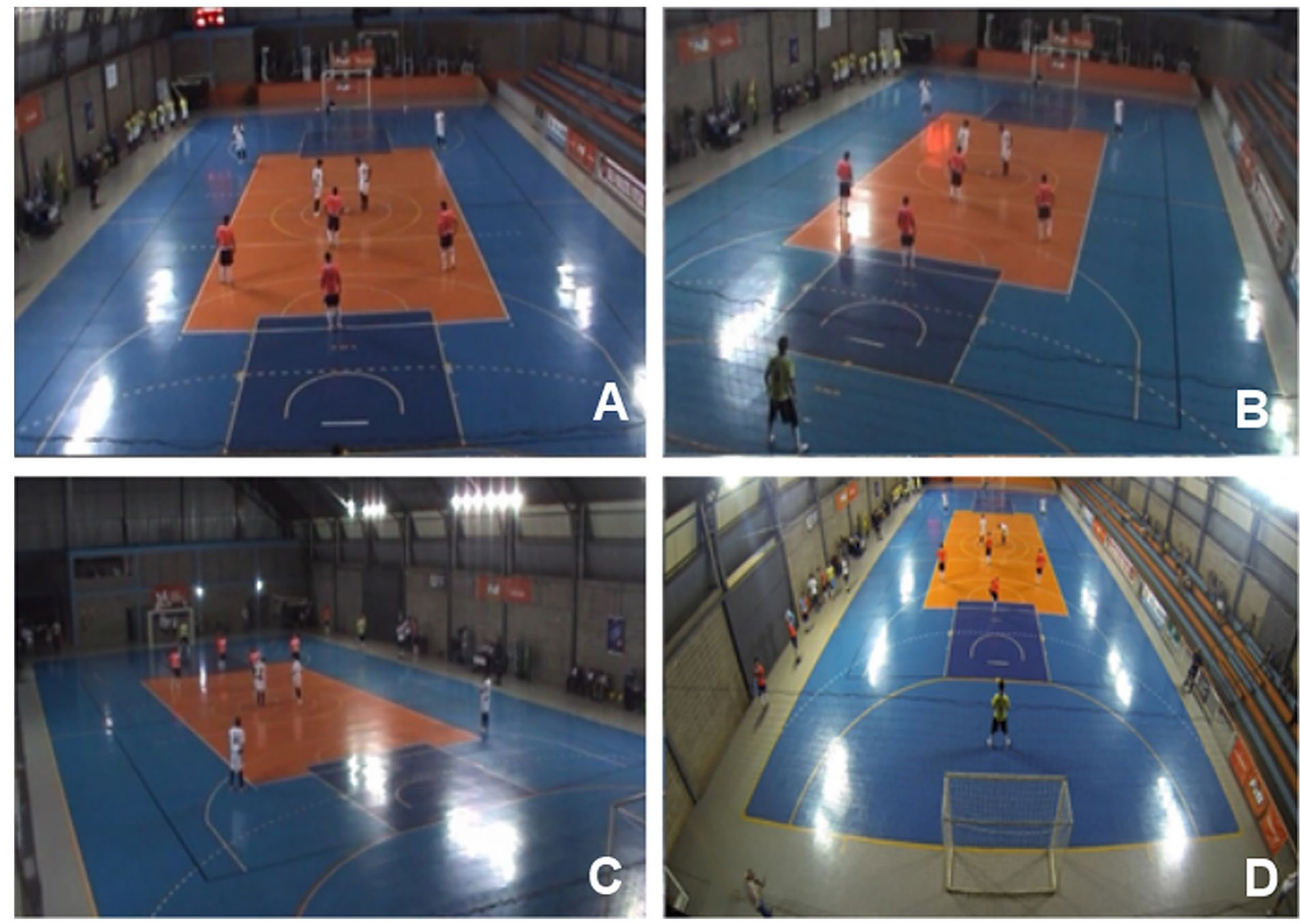

Figure 1. Depiction of how the scene was acquired by the three $C C L$ cameras $(A)-(C)$ and a single CWAL camera (D) and the respective court area that was figured. 
Subsequently, the sequences of images were transferred to a computer in order to perform distortion correction of the wide-angle lens camera images and then perform computational tracking for both types of video.

\section{Radial distortion correction}

Given a distorted point in the original image $\left(x_{d^{\prime}} y_{d}\right)$, the radial lens distortion correction was performed using Equation (1):

$$
\left(\begin{array}{l}
x_{u} \\
y_{u}
\end{array}\right)=\left(\begin{array}{l}
x_{c} \\
y_{c}
\end{array}\right)+L(r)\left(\begin{array}{l}
x_{d}-x_{c} \\
y_{d}-y_{c}
\end{array}\right)
$$

where $x_{c}$ and $y_{c}$ are the distortion centre; and $x_{u}$ and $y_{u}$ are the distortion-free point coordinates.

The shape of the function $L(r)$ determines the amount of distortion correction. For the division model we selected, $L(r)$ can be estimated by considering that $3 \mathrm{D}$ lines in the image must be projected onto 2D straight lines, and minimising the distortion error, which is given by the sum of the squares of the distances from the points to the lines (Alvarez et al. 2009). We applied an automatic method to extract (segment) a set of straight lines within the image; this must be done efficiently, with sub-pixel precision and at low computational cost in order to make the approach reliable for application to real video sequences of interest (Alemán-Flores et al. 2014). The Hough transform (Hough 1959; Duda \& Hart 1972) is a well-known and widely applied method in image processing and computer vision to identify lines in an image. We refer the reader to any computer vision text for a complete description of the method (see for instances, Shapiro \& Stockman 2001; AlemánFlores et al. 2013; Alemán-Flores et al. 2014). For our purposes, the use of Hough classical transform allows easy detection of the lines within an image by the standard related algorithm, which is also quite efficient.

In previous studies (Alemán-Flores et al. 2013, 2014), the proposed algorithm has been described in detail and used for radial distortion correction of single pictures (e.g. paintings, photo of buildings, doors and people walking along the street). In this study, for any video sequence, we first extract an initial collection of distorted lines using the division model with one parameter embedded into the Hough space. It was assumed that the distortion that affected all lines within the image was the same for all of them. Hence, at this stage, a single value for the distortion parameter was estimated for the whole image. The following expression was used (Equation (2)):

$$
L(r)=\frac{1}{1+k_{1} r^{2}}
$$

The selection of longest lines was performed through the usual voting scheme related to the standard Hough method: first we extracted the edge points and estimated the edge point orientations. Each edge point votes for the set of lines in the Hough space with a similar orientation to the edge point orientation. Next, from the voting score matrix, we selected the $n$ most voted lines for each possible value of the radial distortion parameter. This stage provided the set containing the longest lines within the image, which constituted the first step for lens distortion correction. After that, with the same collection of primitives, a two-parameter model was extracted by minimising an energy function given by the distance from the corrected points to their associated lines. If we denote by $d$ the tuple $\left(k_{1}, k_{2}, x_{c^{\prime}} y_{c}\right)$, which defines the distortion model, this minimisation consists of reducing the following energy (Equation (3)):

$$
\left.E(d)=\sum_{j}^{N I} \sum_{i}^{N(j)} \operatorname{distance}\left(C_{d}\left(\bar{x}_{j i}\right) \text {, line }\right)_{j}\right)^{2}
$$

where $\mathrm{NI}$ is the number of lines, $N(j)$ is the number of points of the $j$ th line and $C_{d}\left(\bar{x}_{j i}\right)$ represents the corrected points associated to line ${ }_{i}$, using the model given by $d$. This error measures how distant the points are from their respective lines (Alemán-Flores et al. 2013, 2014). The initial value for $k_{1}$ is given by the final value obtained in the previous step, whereas the initial value for $k_{2}$ is 0 . The centre of distortion is initialised at the geometric centre of the image. The Taylor expansion of $E(d)$ around the initial approximation $d_{0}$ is given by (Equation (4)):

$E(d)=E\left(d_{0}\right)+\nabla\left(E\left(d_{0}\right)\right)^{\top}\left(d-d_{0}\right)+\left(d-d_{0}\right)^{\top} \nabla^{2} E\left(d_{0}\right)\left(d-d_{0}\right)+\cdots$

Since we want to minimise this energy, we derive the previous expression and make it equal to 0 (Equation (5)):

$$
\nabla E(d) \approx \nabla E\left(d_{0}\right)+\nabla^{2} E\left(d_{0}\right)\left(d-d_{0}\right)=0
$$

Note that the minimum of this energy function $E$ stands for the case of the most detected lines that are rectified; so, once this minimum is calculated, the related solution (distortion parameters) is obtained. With the new distortion parameters, an iterative process starts which feedbacks into the initial search of lines using the Hough transform until no new lines are obtained or no more points are added to the existing lines. The iterative optimisation applied provides a refined solution because the most lines are detected, and therefore, the energy function $\mathrm{E}$ handles information for most of the pixels within the image which implies a better distortion correction. By applying a simple mathematical inversion to the model, the image distortion is corrected.

This correction was applied to each frame in the futsal sequence. The computational cost per frame (image resolution was $720 \times 480$ ) was around $30 \mathrm{~ms}$ in an 8-core Intel Xeon (R) CPUX5650 machine with 12.04 Ubuntu Linux. The computational cost for the line extraction stage (only required for the first video frame) was around $5 \mathrm{~s}$. The proposed algorithm to correct the radial distortion can be summarised as follows:

(1) For the first video frame: to extract, in an automatic way, the set of lines using a modified Hough transform embedded with a one-parameter division model. The result of this step is the set of distorted lines and an estimate (initial value) of the first distortion parameter.

(2) For the first video frame: lens distortion model improvement using nonlinear optimisation techniques. The result of this step is an optimised 2-parameter division model and the optimal centre of distortion.

(3) To correct the radial distortion of each video frame using the lens distortion model estimated for the first frame.

We point out that the proposed method is fully automatic and does not require user intervention (Figure 2).

\section{Computational tracking}

Player trajectories were obtained by DVideo ${ }^{\mathrm{TM}}$ software (Barros et al. 2007, 2011). Synchronisation of images was performed 

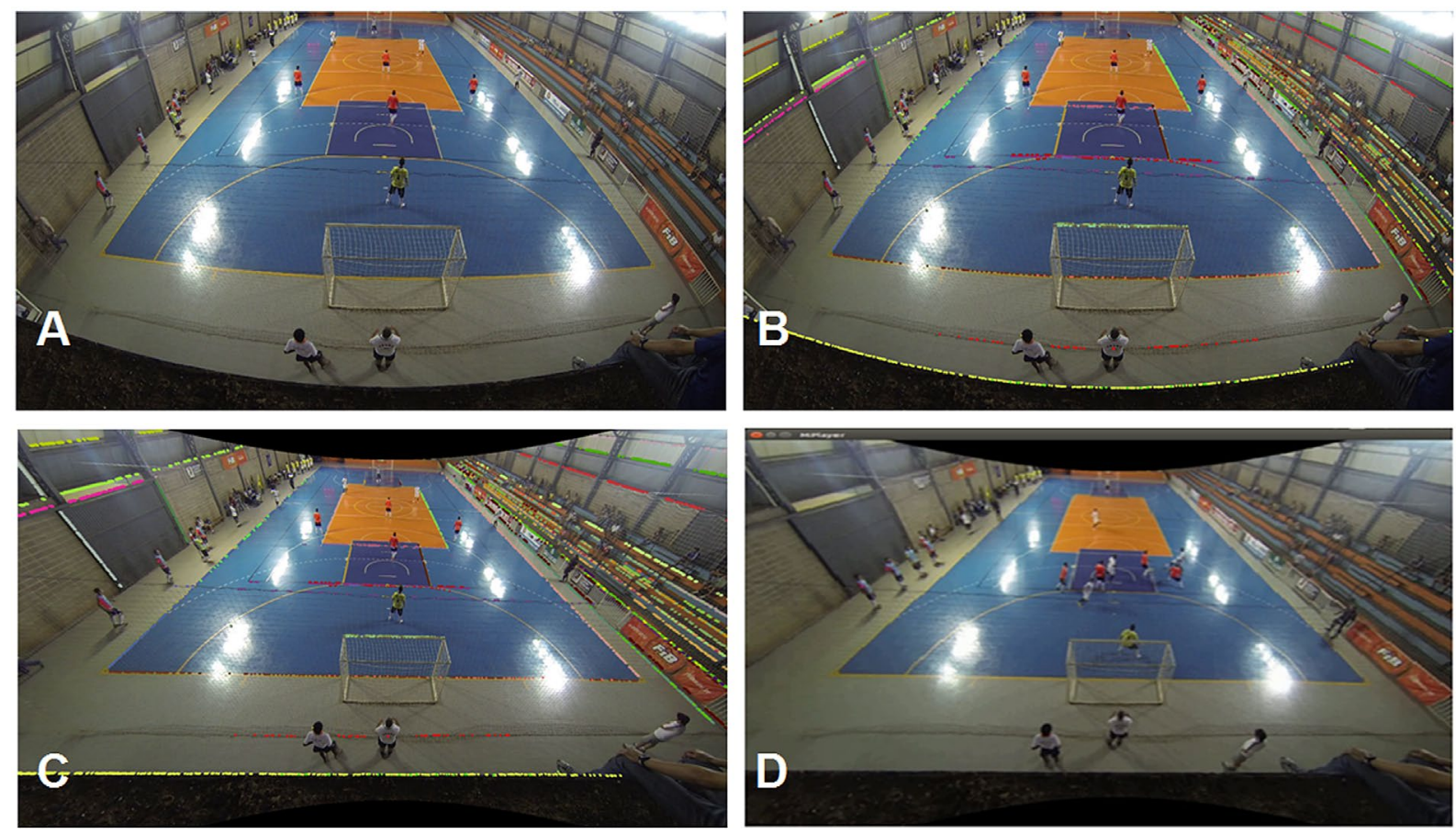

Figure 2. Example of the sequences of steps carried by the proposed algorithm. (i) Loading the original distorted frame of the GoPro ${ }^{\circledR}$ (A), (ii) automatic extraction of the set of lines using a modified Hough transform embedded with a one-parameter division model (B), (iii) lens distortion model improvement using nonlinear optimisation techniques and (C) an example of another corrected frame of the same video sequence (D).

by identifying common events in overlapped regions; then, two-dimensional calibration was done based on 23 control points on the court surface, with real distances previously measured. Game tracking was performed manually frame-by-frame with a mouse by an experienced examiner. The images analysed were taken from the $\mathrm{CCL}$ and a sequence of corrected radial distortion images captured by the CWAL, both from the first $8 \mathrm{~min}$ of the game, which corresponded to 14,400 frames.

After marking the frames, calibration points and player trajectories were reconstituted by means of the direct linear transformation method, which provided player 2D coordinates with respect to court coordinates and compared tracking performed by CCL and CWAL. Data were filtered by a Butterworth third-order low-pass digital filter, with a $0.4 \mathrm{~Hz}$ cut-off frequency. Total distance covered, average velocity and acceleration were calculated in a MATLAB ${ }^{\oplus}$ environment (The MathWorks Inc., USA).

\section{Experimental results}

\section{Assessment of uncertainties in measurements - calibration}

In order to measure the uncertainties related to the measurement of calibration points, the 23 calibration points on the court surface were measured manually, 10 times each, by an experienced examiner, and reconstructed for the CWAL camera with corrected image sequences. The results were compared to actual expected values obtained by direct measurement, as proposed by Barros et al. (2011) that is, the mean Euclidean distances were calculated between actual values obtained by direct measurement and reconstructed values from the court surface points after marking. We found a mean uncertainty of $0.19 \mathrm{~m}$. Figure 3 shows the uncertainties related to the measurements of each point.

\section{Assessment of trajectories obtained by tracking during the game}

As a means of assessing whether player trajectories tracked by CCL and CWAL sequences were similar, statistical analysis of cross-correlation with a zero lag was applied to assess the similarity between the coordinates ( $x$ and $y$ ) of the motion vector in every time series. For this procedure, a specific cross-correlation routine was used (function 'xcorr.m'), which is available in the $M A T L A B^{\oplus}$ statistical package. In order to find the magnitude of the actual difference between measurements made, we also calculated the Euclidean distance between the $2 \mathrm{D}$ reconstructed coordinates for both types of tracking, in each frame analysed.

The correlation analysis showed that tracking performed with image sequences from corrected CCL and CWAL presented basically very similar $x$ and $y$ coordinates ( $r=0.99$ for both). This is evident in the graphic display of these coordinates over time for one of the players (Figure 4).

Table 1 includes error magnitude data associated with tracking of each player performed with CWAL corrected image sequences, compared to tracking performed with CCL by means of calculation of the Euclidean distance between coordinates of both types of tracking.

\section{Results for distances covered by players during a futsal game}

Table 2 presents the results for total distance covered in $5 \mathrm{~min}$ and compares tracking performed by CCL and CWAL image sequences.

\section{Uncertainties associated with DVideo tracking, performed automatically}

In order to assess the accuracy of measurements of automatic tracking by segmentation in $\mathrm{DVideo}^{\mathrm{TM}}$ software 


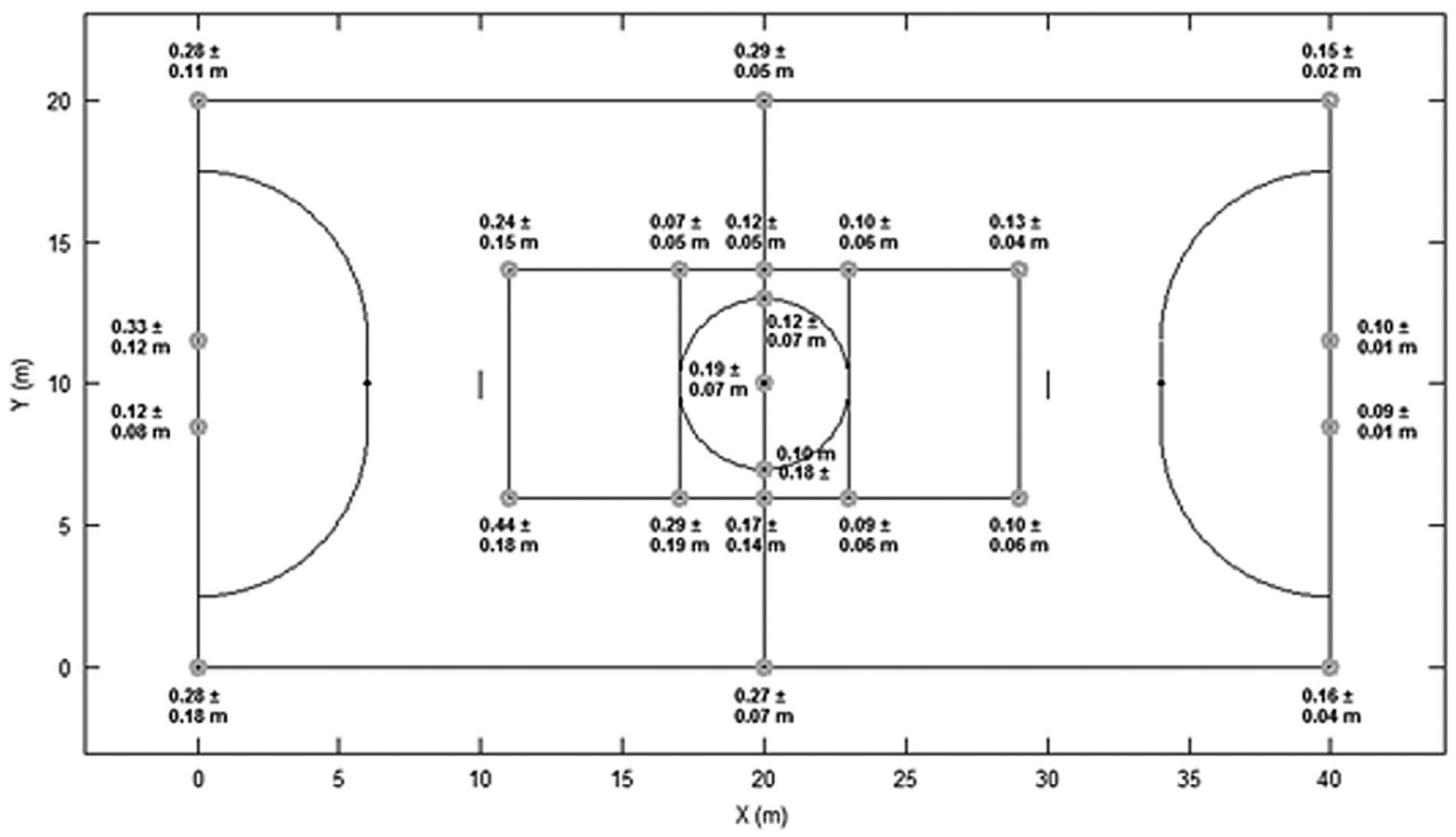

Figure 3. Illustration of the points used for calibration and assessment of uncertainties related to reconstruction of static points on the court surface. Mean values \pm standard deviation.
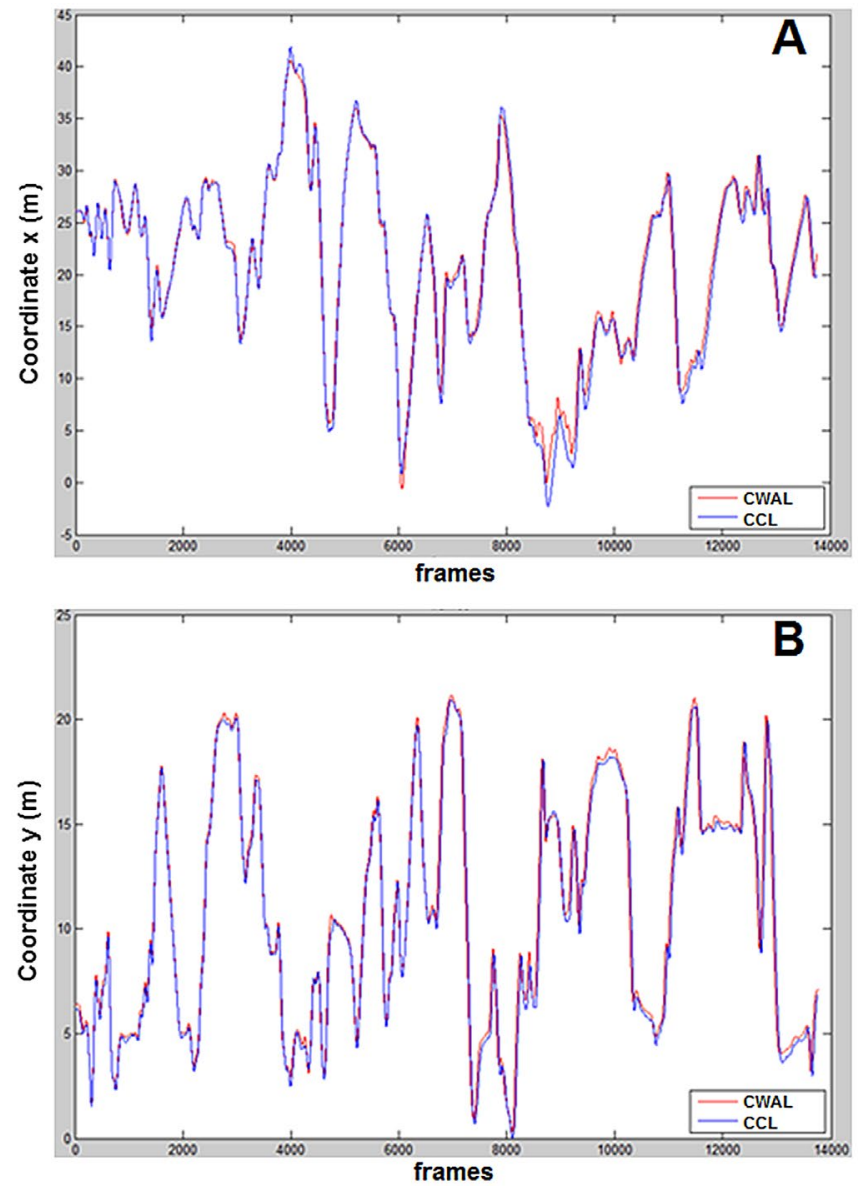

Figure 4. Motion of one player in $x$-coordinate (A) and $y$-coordinate (B) in 14,000 frames analysed during the official match, by tracking with CWAL and CCL video sequences. 
Table 1. Values for Euclidean distance $(\mathrm{m})$ between 2D points reconstructed in each frame, of all players' tracking performed with CCL and CWAL.

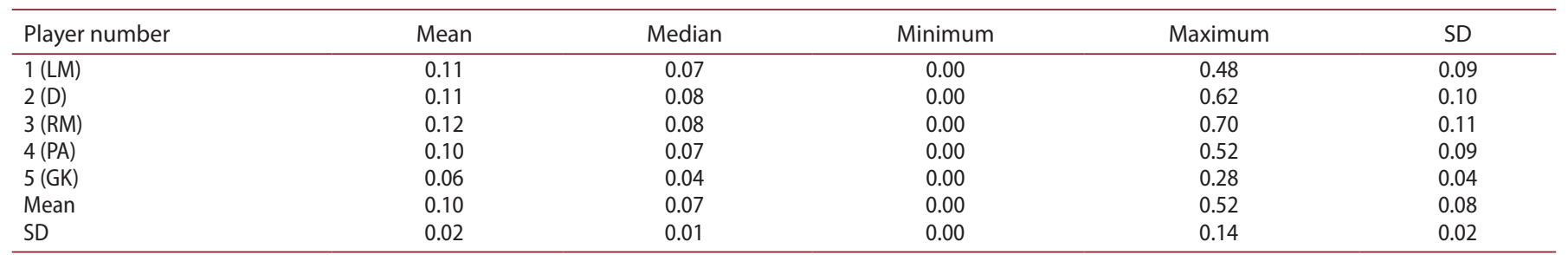

Notes: $\mathrm{SD}=$ standard deviation, $\mathrm{LM}=$ left midfielder, $\mathrm{D}=$ defender, $\mathrm{RM}=$ right midfielder, $\mathrm{PA}=$ pivot forwarder, $\mathrm{GK}=$ goalkeeper.

Table 2. Distance covered $(\mathrm{m})$ by five players during the first $5 \mathrm{~min}$ of the game, calculated with data obtained by tracking performed with image sequences taken from CCL and CWAL with corrected sequences.

\begin{tabular}{lccc}
\hline & & Distance covered $(\mathrm{m})$ & \\
\cline { 2 - 4 } Player number & $\mathrm{CCL}$ & $\mathrm{CWAL}$ & Diff $(\%)$ \\
\hline $1(\mathrm{LM})$ & 555.50 & 545.39 & -1.82 \\
2 (D) & 463.05 & 450.61 & -2.69 \\
3 (RM) & 584.06 & 565.50 & -3.18 \\
4 (PA) & 488.52 & 476.45 & -2.47 \\
5 (GK) & 156.58 & 156.89 & +0.20 \\
Mean & 449.54 & 438.97 & 2.11 \\
SD & 170.92 & 164.65 & \\
\hline
\end{tabular}

Notes: Diff = percentage difference, $\mathrm{SD}=$ standard deviation, $\mathrm{LM}=$ left midfielder, $\mathrm{D}=$ defender, $\mathrm{RM}=$ right midfielder, $\mathrm{PA}=$ pivot forwarder, $\mathrm{GK}=$ goalkeeper.
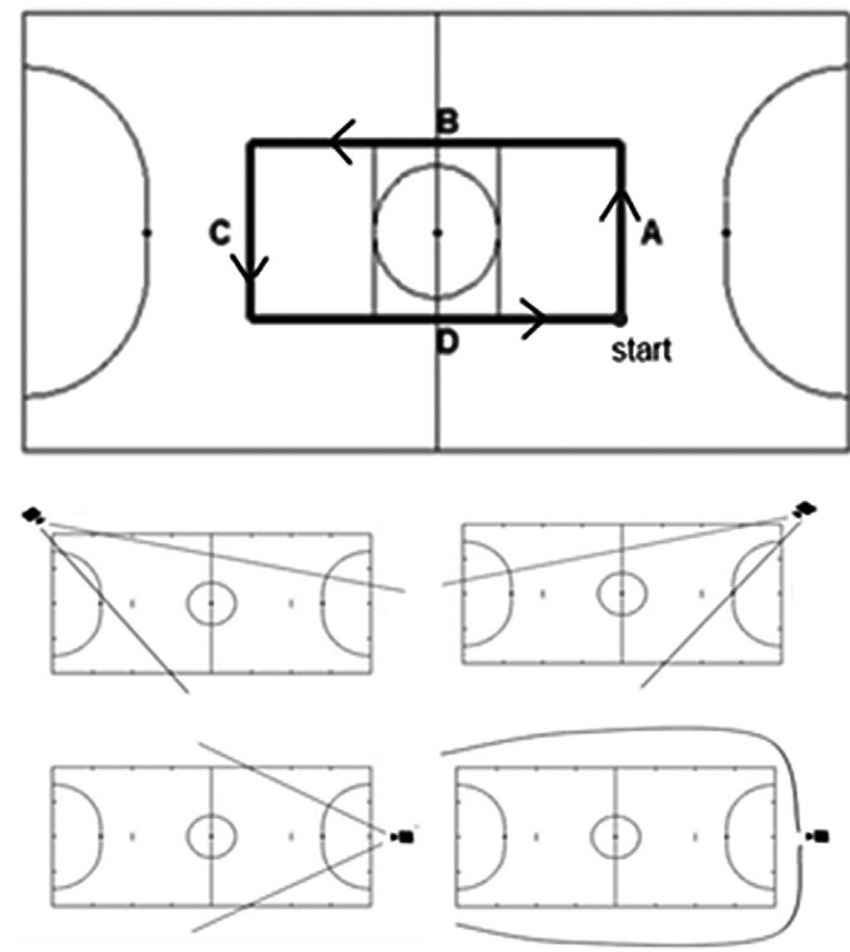

Figure 5. Illustration of the trajectory defined for the experiment on automatic tracking in a controlled environment and the position of cameras, similar to that adopted to record the game. $A=C=9 \mathrm{~m} . B=D=18 \mathrm{~m}$.

(Figueroa et al. 2006; Barros et al. 2011), we conducted an experiment in a controlled environment, in a set-up similar to the recommendations of Pers et al. (2002) (experiment II). One of the players was selected and instructed to move across certain distances on the court, using a predefined route, for a total of 10 laps (Figure 5).

We obtained automatic tracking of $89.01 \%$ of the processed frames in this experiment. The total distance calculated experimentally was $535.60 \mathrm{~m}$ vs. the actual circuit distance of $540 \mathrm{~m}$, which represented a relative error of $0.81 \%$.

\section{Results of player mean velocity, acceleration and trajectories}

Figure 6 represents the average velocity and Figure 7 the acceleration of one player analysed obtained at each point in time during $5 \mathrm{~min}$ of the game. Figure 8 shows the trajectories of all players during 8 min of tracking, comparing CWAL and CCL.

\section{Root-mean-square error}

The values of variables of interest obtained by tracking performed with a GoPro ${ }^{\oplus}$ camera (CWAL) were statistically 

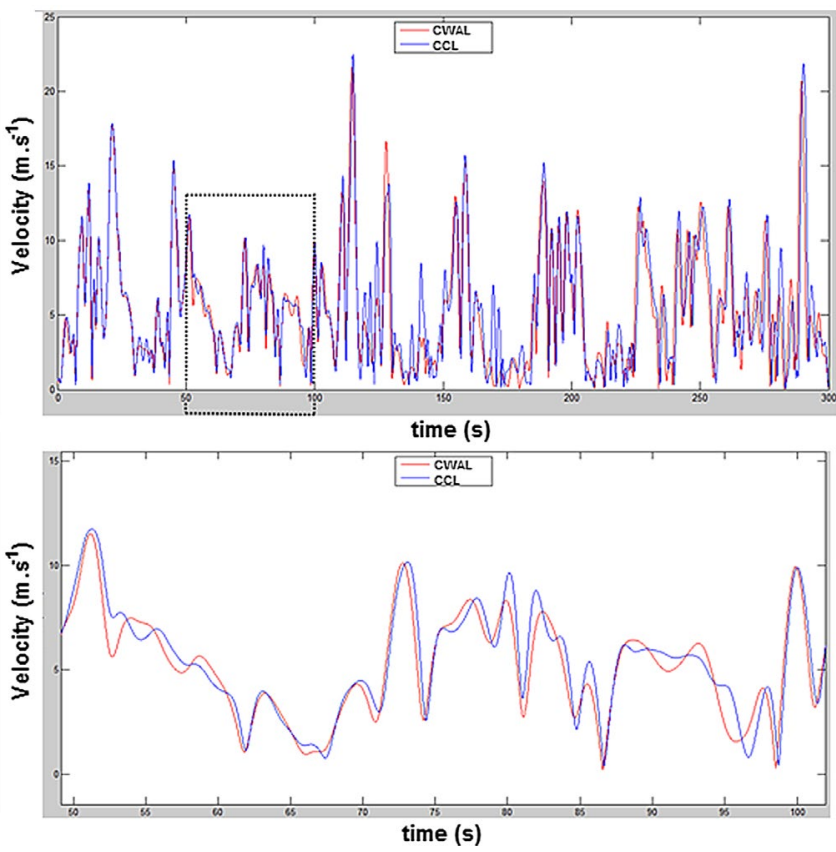

Figure 6. Graphical plot of one player's velocity in temporal series (5 min) and an enlarged excerpt (50 s), during the official match, by tracking with CWAL and CCL video sequences.
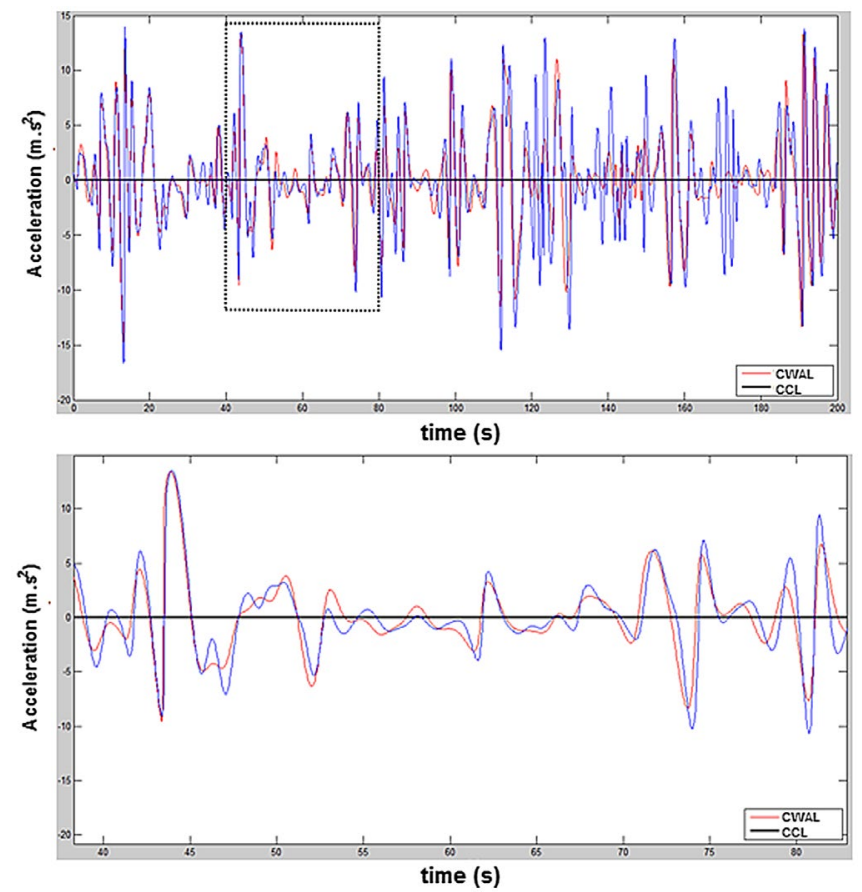

Figure 7. Graphical plot of one player's acceleration in temporal series ( $3 \mathrm{~min})$ and an enlarged excerpt $(40 \mathrm{~s})$, during the official match, by tracking with CWAL and CCL video sequences.

processed as a way to assess root-mean-square (RMS) error, following the recommendations of Pers et al. (2002) and considering Equation (6).

$$
\mathrm{RMS}=\sqrt{\frac{1}{n} \sum_{i=1}^{n}\left|M_{\mathrm{EXP}}-M_{\mathrm{REF}}\right|^{2}}
$$

where RMS = root-mean-square error; $n=$ number of analysed frames; $i=$ current frame; $M_{\mathrm{EXP}}=$ experimental measurement value; $M_{\mathrm{REF}}=$ reference value, in frame $i$.

The selected variables were two-dimensional position (P2-D) reconstructed and velocity (VEL). Tables 3 and 4 contain the results of RMS error calculations for P2-D and VEL, respectively: in the calibration experiment, for tracking during the game (manual tracking) and in the automatic tracking experiment. 
CCL
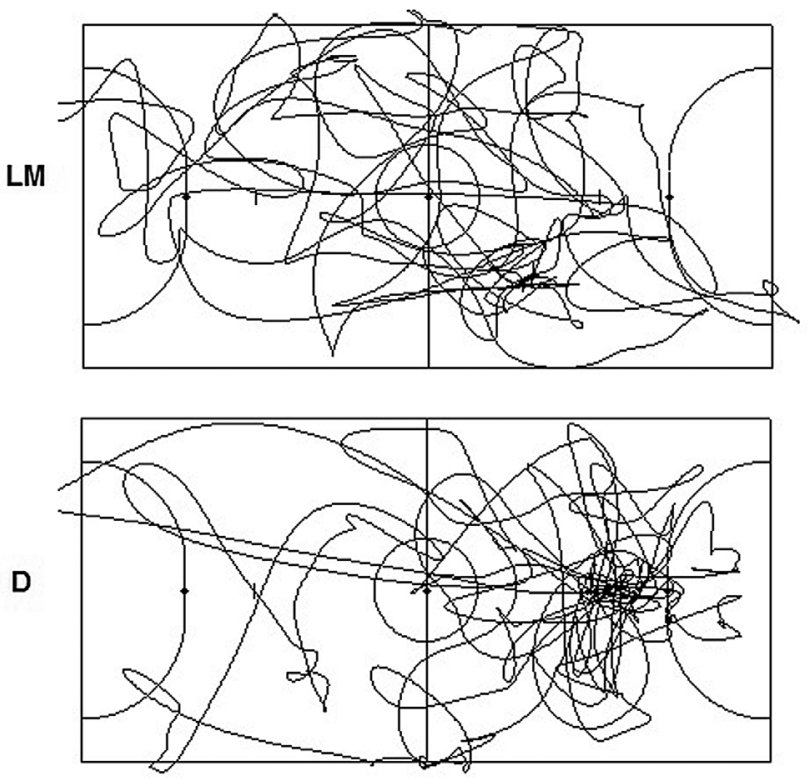

RM
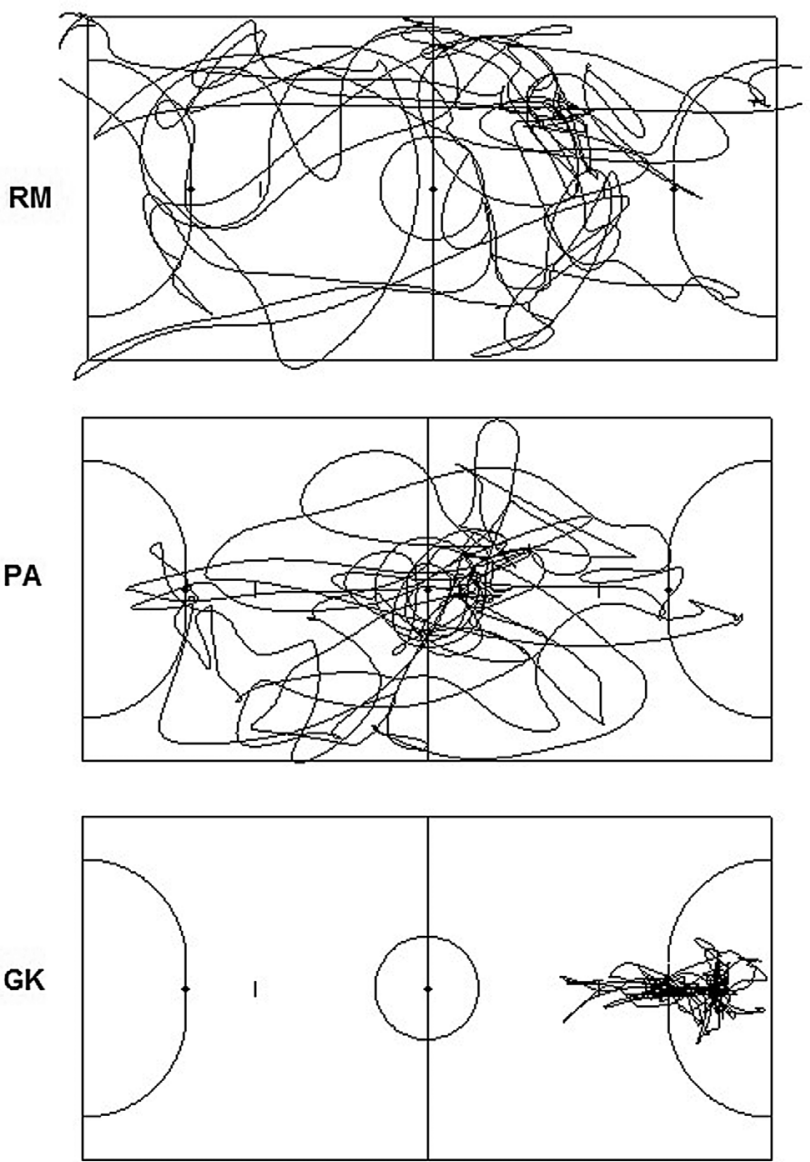

CWAL
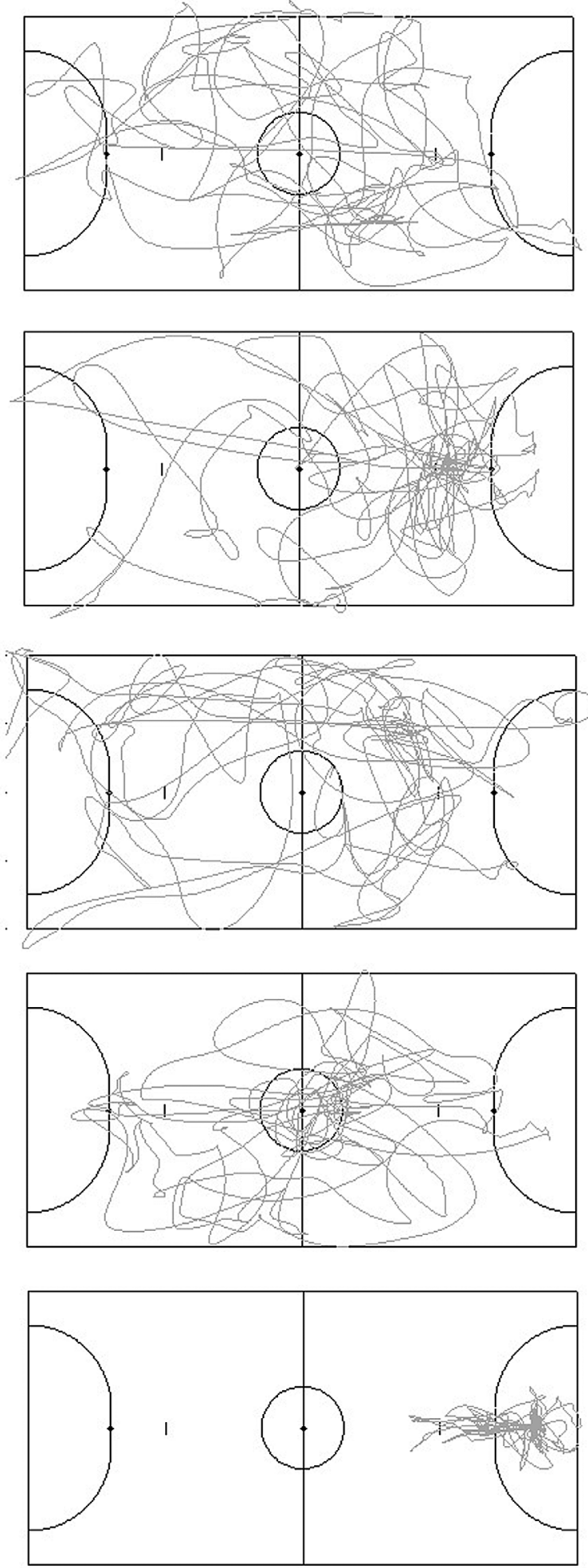

Figure 8. Trajectories of futsal players for eight minutes during the first half-time $(20 \mathrm{~min})$. GK= goalkeeper. $\mathrm{LM}=$ left midfielder. $\mathrm{D}=$ defender. $\mathrm{RM}=$ right midfielder. $\mathrm{PA}=$ pivot forwarder. Defence on the right side.

Table 3. RMS error values of the calibration experiment, of the 8 -min tracking of the game $(14,400$ frames $\times 5$ players $=72,000$ frames $)$ and the automatic tracking experiment ( $4 \mathrm{~min}=7200$ frames) for P2D, in $\mathrm{m}$.

\begin{tabular}{lccc}
\hline RMS error & Our proposal & Pers et al. (2002) & Vučkovič et al. (2010) \\
\hline Calibration experiment & 0.23 & 0.2 & $0.09-0.34$ \\
Tracking of game & 1.29 & $0.27-0.64$ & $0.11-0.42$ \\
Automatic tracking experiment & 0.32 & $0.25-0.40$ & $0.17-0.50$ \\
\hline
\end{tabular}


Table 4. RMS error values of the calibration experiment, of the 8-min tracking of the game $(14,400$ frames $\times 5$ players $=72,000$ frames $)$ and the automatic tracking experiment ( $4 \mathrm{~min}=7200$ frames) for VEL, in $\mathrm{m} \mathrm{s}^{-1}$.

\begin{tabular}{|c|c|c|c|}
\hline RMS error & Our proposal & Pers et al. (2002) & Vučkovič et al. (2010) \\
\hline Tracking of game & 0.87 & $0.04-2.10$ & $0.50-0.58$ \\
\hline Automatic tracking experiment & 0.71 & $0.21-0.35$ & $0.16-0.74$ \\
\hline
\end{tabular}

Table 5. Reference values of the tests performed.

\begin{tabular}{lcc}
\hline & & Reference values \\
\cline { 2 - 3 } Experiment & PD actual coordinates obtained by direct measurement (Figure 4) & VEL \\
\hline Calibration experiment $(n=23$ static points) & 2D actual coordinates of court lines (Figure 3) & Values obtained by tracking performed with \\
Automatic tracking experiment $(n=1$ player) & 2D coordinates of game tracking with CCL \\
Game tracking ( $n=5$ players) & & Values obtained by tracking performed with \\
\hline
\end{tabular}

Our experiments were based on the work of Pers et al. (2002). Our calibration experiment was similar to one that involved the choice of static position of players on the court. Game tracking was similar to Experiment I, whereas automatic tracking that involved movements across court lines was similar to experiments II and III (see Pers et al. 2002).

\section{Discussion}

The objective of this study was to assess the applicability and reliability of a single wide-angle lens camera for the kinematic analysis of tracking in futsal. The advantages of this model are that this camera has a field of view that is able to capture the whole scene, with no need of auxiliary cameras, which consequently reduces financial and material costs of footage analysis.

The GoPro ${ }^{\circledR}$ model HERO3+ (GoPro 2014) is a small camera $(59 \times 21 \times 41 \mathrm{~mm})$ with high definition video recording. It can be activated by remote control, weighs $0.74 \mathrm{~kg}$, and has Wi-Fi and a $170^{\circ}$ field of view (GoPro 2014; Kim et al. 2014). Its use was only recently introduced in the assessment of sport performance from the biomechanics approach, especially in kinematic studies of human motion in sprint running (Gasser et al. 2013; Born et al. 2014), in an aquatic environment, in order to identify the kinematic pattern of the foot during a treadmill run, on the floor and in the water (Hoover 2014), or very close to the water, as in the investigation of sailor performance (Pluijms et al. 2014).

Clemente et al. (2014) used a single GoPro ${ }^{\circledR}$ for tracking soccer players; however, they only partially reported the tracking error values and the model of distortion correction. We compared our experimental result errors with figures available in the literature. As for the calibration experiment and the reconstruction of static positions on the court with previously measured distances in relation to the starting point, our results were in line with the literature. Barros et al. (2011), using the DVideo ${ }^{\mathrm{TM}}$ system, found a mean uncertainty of $0.20 \mathrm{~m}$ in the definition of positions on the court; in our study, the mean uncertainty was similar, at $0.19 \mathrm{~m}$.

Our results for the experiment with movements across court lines (in a controlled environment) were close to those found by Pers et al. (2002), which used two CWALs for tracking handball, and to the study by Vučkovič et al. (2010), which used a CWAL for tracking squash (Tables 4 and 5). However, P2-D had a greater RMS error value in our study, regarding game tracking, when we specified that the reference values would be those found with $\mathrm{CCL}$ tracking. In that sense, we must highlight we determined this error using the difference between the values obtained from the CCL and CWAL tracking in actual game conditions, which was not the case for previous studies. We should also point out that the game situation increased visual difficulties in following each player, due to the complexity of some situations, such as mutual occlusion (Figueroa et al. 2006), which could limit results comparison in part of this tracking experiment. Pers et al. (2002) conducted their experiments in controlled conditions, with predefined trajectories and velocity (reference values).

When using a single camera, it promotes a more severe mutual occlusion effect. In the past, some studies (Barros et al. 2007, 2011) suggested that a solution to increase the percentage of automatic tracking and reduce the mutual occlusion effects on the frames analysed by tracking in sports context would require placing cameras on both sides of the game (i.e. multi-camera set-up). However, an increase in processing time is expected (Barros et al. 2007) due to increase in time to calibration, synchronisation and segmentation phases in all sequences of images from all cameras. In addition, there are severe restrictions on where you can place your cameras in official sports events (Barros et al. 2011). In that sense, the single wide-angle lens camera set-up seems to be quite advantageous by eliminating the'synchronization'step, reducing the number of cameras (i.e. financial cost) and evaluators responsible for cameras, reducing the time in calibration and segmentation, and finally reducing the computational cost.

After analysis of displacement curves on the $x$ and $y$ axes (Figure 4), we found a high similarity between the reconstructed coordinates of tracking performed with corrected distortion images from CCL and CWAL cameras (Table 1), which was confirmed by the applied analysis of temporal correlations and low RMS error values obtained.

Furthermore, in practical terms, distances covered during the first 5 min of the game calculated with tracking performed by CCL (449.54 $\pm 170.92 \mathrm{~m})$ and CWAL (438.97 $\pm 164.65 \mathrm{~m})$ presented a mean variation of $2.11 \%\left(2.11 \mathrm{~m} \mathrm{~min}^{-1}\right)$. Pers et al. (2002) reported an error between 10 and $6 \mathrm{~m} \mathrm{~min}^{-1}$ regarding total distances covered by players, and these values were greater than those found in our study. We have to consider that minor variations existed regarding different measurements of this phenomenon (i.e. frame markings), done by the same examiner at different times, to compare tracking performed with CCL and CWAL. In addition, the highest value for the Euclidean distance found for 2D tracking coordinates in CWAL and CCL without data filtering was $0.7 \mathrm{~m}$, 
with a mean value of $0.09 \mathrm{~m}$ for all players (Table 1 ). These results allow us to say that the error was acceptable, and the method adopted for radial distortion correction had reasonable accuracy.

Other approaches to radial distortion correction with reasonable accuracy have involved placing a chessboard close to the camera (Rossi et al. 2013; Silvatti et al. 2013). However, for tracking analysis, the use of chessboards is difficult, as cameras are usually fixed to posts or higher spots around the court so they can frame the largest area possible (as in Pers et al. 2002; Perše et al. 2009; Moura et al. 2012; Bueno et al. 2014).

Automatic tracking in DVideo ${ }^{\mathrm{TM}}$ with CWAL had a relative error percentage of $0.81 \%$ for determining the total distance covered, when comparing the actual value (distance known) to the experimental value obtained in a controlled environment. Bueno et al. (2014) reported a similar value (0.8\%) when they determined distances covered in futsal with the use of DVideo ${ }^{\mathrm{TM}}$, which also indicates that the use of $\mathrm{a} \mathrm{GoPro}^{\circledR}$ with corrected radial distortion is equivalent to the use of other CCL.

\section{Conclusion}

This study aimed to test a radial distortion correction algorithm applied to sequences of images obtained during an official professional futsal match, using a single camera with wide-angle lens for computational tracking purposes. To the extent of our knowledge, this was the first study in the field of futsal that made use of a single camera of this type to investigate kinematics of players' trajectories, describing the error assessment with the method adopted.

The results show that the use of image sequences with corrected radial distortion of a single wide-angle lens camera, such as the $\mathrm{GoPro}^{\circledR} \mathrm{HERO} 3+$, using Hough transform to detect highly distorted lines in the image, by means of an automatic detection algorithm of sub-pixel borders precision (Alemán-Flores et al. 2014), is a highly applicable and reliable way to conduct kinematics investigation of trajectories and distances covered by futsal players. In addition, the results also indicate that the use of this camera with radial distortion corrected is similar to a conventional multi-camera set-up without severe lens distortion.

Future research should implement algorithms to optimise automatic tracking in competitive situations with image sequences taken from a single camera like $\mathrm{GoPro}^{\circledR}$, which is a condition that has lower costs of image storage (i.e. a single camera), and the smallest number of human interventions (i.e. automatic tracking) and thus lower processing time.

\section{Acknowledgements}

The authors would like to thank Filipe Gonçalves Mesquita for his technical support.

\section{Disclosure statement}

No potential conflict of interest was reported by the authors.

\section{Funding}

This work was supported by FAPESP [2013/08302-6] and PIBIC/USP [14.1.101.90.2].

\section{References}

Alemán-Flores M, Alvarez L, Gomez L, Santana-Cedrés D. 2013. Wide-angle lens distortion correction using division models. In: Ruiz-Shulcloper $J$, di Baja GS, editors. Progress in pattern recognition, image analysis, computer vision, and applications. Berlin: Springer; p. 415-422.
Alemán-Flores M, Alvarez L, Gomez L, Santana-Cedrés D. 2014. Line detection in images showing significant lens distortion and application to distortion correction. Pattern Recognit Lett. 36:261-271.

Alvarez L, Gomez L, Sendra JR. 2009. An algebraic approach to lens distortion by line rectification. J Math Imaging Vis. 35:36-50.

Barbero-Alvarez JC, Soto VM, Barbero-Alvarez V, Granda-Vera J. 2008. Match analysis and heart rate of futsal players during competition. J Sports Sci. 26:63-73.

Barros RML, Misuta MS, Menezes RP, Figueroa PJ, Moura FA, Cunha SA, Anido R, Leite NJ. 2007. Analysis of the distances covered by first division Brazilian soccer players obtained with an automatic tracking method. J Sports Sci Med. 6:233-242.

Barros RML, Menezes RP, Russomanno TG, Misuta MS, Brandão BC, Figueroa PJ, Leite NJ, Goldenstein SK. 2011. Measuring handball players trajectories using an automatically trained boosting algorithm. Comput Methods Biomech Biomed Eng. 14:53-63.

Born D-P, Holmberg H-C, Goernert F, Sperlich B. 2014. A novel compression garment with adhesive silicone stripes improves repeated sprint performance - a multi-experimental approach on the underlying mechanisms. BMC Sports Sci Med Rehabil. 6:1-9.

Bueno MJO, Caetano FG, Pereira TJ, De Souza NM, Moreira GD, Nakamura FY, Cunha SA, Moura FA. 2014. Analysis of the distance covered by Brazilian professional futsal players during official matches. Sports Biomech. 13:230-240.

Carling C, Bloomfield J, Nelsen L, Reilly T. 2008. The role of motion analysis in elite soccer: contemporary performance measurement techniques and work rate data. Sports Med. 38:839-862.

Clemente FM, Couceiro MS, Martins FM, Figueiredo AJ, Mendes RM. 2014. Análise de jogo no Futebol: Métricas de avaliação do comportamento coletivo [Match analysis on football: metrics to evaluate the collective behavior]. Motri. 10:14-26.

Duda RO, Hart PE. 1972. Use of the Hough transformation to detect lines and curves in pictures. Commun ACM. 15:11-15.

FIFA. 2014. Futsal laws of the game 2012/2013 [Internet]. Zurich: Fédération Internationale de Football Association; [cited 2014 Nov 12]. Available from: http://www.fifa.com/mm/document/affederation/generic/51/44/50/ futsallawsofthegameen.pdf.

Figueroa PJ, Leite NJ, Barros RML. 2006. Background recovering in outdoor image sequences: an example of soccer players segmentation. Image Vis Comput. 24:363-374.

Gasser M, Habegger B, Goette J, Jacomet M. 2013. Feature extraction from biological motion with PARTwear: an application for sprint running. Paper presented at: 3rd International Conference on Ambulatory Monitoring of Physical Activity and Movement; Amherst, USA.

GoPro. 2014. Hero3+ Black [Internet]. [cited 2014 Nov 12]. Available from: http://gopro.com/cameras/hd-hero3-black-edition.

Hoover J. 2014. Differences in strike index between land treadmill and aquatic treadmill running in experienced distance runners. Paper presented at: Graduate Research Symposium of the Utah State University - Paper 45; Logan, USA.

Hough PVC. 1959. Machine analysis of bubble chamber pictures. Paper presented at: International Conference on High Energy Accelerators and Instrumentation; CERN Switzerland/France.

Kim JH, Pyeon MW, Eo YD, Jang IW. 2014. An experiment of three-dimensional point clouds using GoPro. Int J Civ Archit Struct Constr Eng. 8:82-85.

Martínez-Gallego R, Guzmán JF, James N, Pers J, Ramón-Llin J, Vuckovic G. 2013. Movement characteristics of elite tennis players on hard courts with respect to the direction of ground strokes. J Sports Sci Med. 12:275-281.

Morais E, Ferreira A, Cunha SA, Barros RML, Rocha A, Goldenstein S. 2014. A multiple camera methodology for automatic localization and tracking of futsal players. Pattern Recognit Lett. 39:21-30.

Moura FA, Santana JE, Marche AL, Aguiar TH, Rodrigues ACMA, Barros RML, Cunha SA. 2012. Quantitative analysis of futsal players organization on the court. Rev Port Cien Desp. 11:105-108.

Pers J, Bon M, Kovacic S, Sibila M, Dezman B. 2002. Observation and analysis of large-scale human motion. Human Mov Sci. 21:295-311.

Pers J, Kovacic S. 2002. Nonparametric, model-based radial lens distortion correction using tilted camera assumption. Paper presented at: Computer Vision Winter Workshop; Bad Aussee, Austria. 
Pers J, Kristan M, Perse M, Kovacic S. 2008. Analysis of player motion in sports matches. Paper presented at: Dagstuhl Seminar Proceedings - Computer Science in Sport - Mission and Methods; Dagstuhl, Germany.

Perše M, Kristan M, Kovačič S, Vučkovič G, Perš J. 2009. A trajectory-based analysis of coordinated team activity in a basketball game. Comput Vis Image Und. 113:612-621.

Pluijms JP, Canal-Bruland R, Hoozemans MJ, Savelsbergh GJ. 2014. Visual search, movement behaviour and boat control during the windward mark rounding in sailing. J Sports Sci. 33:398-410.

Rossi MM, Silvatti AP, Dias FA, Barros RM. 2013. Improved accuracy in 3D analysis using DLT after lens distortion correction. Comput Methods Biomech Biomed Eng. 18:993-1002.

Shapiro L, Stockman G. 2001. Computer vision. New Jersey (NJ): Prentice Hall.
Silvatti AP, Cerveri P, Telles T, Dias FA, Baroni G, Barros RM. 2013. Quantitative underwater $3 \mathrm{D}$ motion analysis using submerged video cameras: accuracy analysis and trajectory reconstruction. Comput Methods Biomech Biomed Eng. 16:1240-1248.

Toki S, Sakurai S. 2005. Quantitative match analysis of soccer games with two dimensional DLT procedures. Paper presented at: 20th Congress of International Society of Biomechanics; Cleaveland, USA.

Vilar L, Araujo D, Davids K, Travassos B, Duarte R, Parreira J. 2014. Interpersonal coordination tendencies supporting the creation/prevention of goal scoring opportunities in futsal. Eur J Sport Sci. 14:28-35.

Vučkovič G, Pers J, James N, Hughes M. 2010. Measurement error associated with the SAGIT/Squash computer tracking software. Eur J Sport Sci. 10:129-140. 